\title{
Prognostic Factors for Long-Term Survival in Patients with Ampullary Carcinoma: The Results of a 15-Year Observation Period after Pancreaticoduodenectomy
}

\author{
Fritz Klein, ${ }^{1}$ Dietmar Jacob, ${ }^{2}$ Marcus Bahra, ${ }^{1}$ Uwe Pelzer, ${ }^{3}$ Gero Puhl, ${ }^{1}$ \\ Alexander Krannich, ${ }^{4}$ Andreas Andreou, ${ }^{1}$ Safak Gül, ${ }^{1}$ and Olaf Guckelberger ${ }^{1}$ \\ ${ }^{1}$ Department of General, Visceral, and Transplantation Surgery, Charité Campus Virchow Universitätsmedizin Berlin, \\ 13353 Berlin, Germany \\ ${ }^{2}$ Department of General and Visceral Surgery, Bielefeld Evangelical Hospital, 33617 Bielefeld, Germany \\ ${ }^{3}$ Department of Hematology/Oncology, Comprehensive Cancer Center, Charité Universitätsmedizin Berlin, 13353 Berlin, Germany \\ ${ }^{4}$ Department of Biostatistics, Coordination Center for Clinical Trials, Charité Universitätsmedizin Berlin, 13353 Berlin, Germany
}

Correspondence should be addressed to Fritz Klein; fritz.klein@charite.de

Received 15 October 2013; Accepted 14 January 2014; Published 2 March 2014

Academic Editor: Attila Olah

Copyright (C) 2014 Fritz Klein et al. This is an open access article distributed under the Creative Commons Attribution License, which permits unrestricted use, distribution, and reproduction in any medium, provided the original work is properly cited.

\begin{abstract}
Introduction. Although ampullary carcinoma has the best prognosis among all periampullary carcinomas, its long-term survival remains low. Prognostic factors are only available for a period of 10 years after pancreaticoduodenectomy. The aim of this retrospective study was to identify factors that influence the long-term patient survival over a 15-year observation period. Methods. From 1992 to 2007, 143 patients with ampullary carcinoma underwent pancreatic resection. 86 patients underwent pyloruspreserving pancreaticoduodenectomy $(60 \%)$ and 57 patients underwent standard Kausch-Whipple pancreaticoduodenectomy (40\%). Results. The overall 1-, 5-, 10-, and 15-year survival rates were $79 \%, 40 \%, 24 \%$, and 10\%, respectively. Within a mean observation period of $30(0-205)$ months, $100(69 \%)$ patients died. Survival analysis showed that positive lymph node involvement $(P=0.001)$, lymphatic vessel invasion $(P=0.0001)$, intraoperative administration of packed red blood cells $(P=0.03)$, an elevated CA 19-9 $(P=0.03)$, jaundice $(P=0.04)$, and an impaired patient condition $(P=0.01)$ are strong negative predictors for a reduced patient survival. Conclusions. Patients with ampullary carcinoma have distinctly better long-term survival than patients with pancreatic adenocarcinoma. Long-term survival depends strongly on lymphatic nodal and vessel involvement. Moreover, a preoperative elevated CA 19-9 proved to be a significant prognostic factor. Adjuvant therapy may be essential in patients with this risk constellation.
\end{abstract}

\section{Introduction}

Ampullary carcinomas arise from the ampulla or papilla of Vater (the duodenal papilla) and account for $0.2 \%$ of tumors of the gastrointestinal tract. However, with a proportion of $7 \%$ to $9 \%$, they represent the second largest proportion (after pancreatic carcinoma) of periampullary carcinomas, which include ampullary carcinomas and carcinomas of the pancreas, the distal bile duct, and the periampullary duodenum [1-3]. In contrast with other carcinomas of the periampullary region, ampullary carcinomas have a higher resection rate, a lower recurrence rate, and a better overall prognosis [3-6].
To date, the etiology of ampullary carcinoma has not been clearly identified. An adenoma-to-carcinoma sequence similar to that of colon carcinoma has been described for ampullary carcinoma [7].

In traditional terms, ampullary cancer is already distinguished from carcinomas of the pancreas, bile duct, and duodenum. For one thing, due to their anatomical location, ampullary tumors become clinically apparent early because of bile or pancreatic duct occlusion [8]. Thus, ampullary carcinomas are often diagnosed at an early tumor stage and, therefore, have a higher probability of successful surgical resection [4]. Secondly, the 5-year survival rate is reported 
with up to $39 \%$, which is between that of duodenal carcinoma (59\%) and carcinomas of the pancreas or bile duct $(15 \%$ and $27 \%$, resp.) $[3,9,10]$. A reason for the better overall prognosis may be the difference in the histological origin of ampullary carcinomas. As early as 1963, Whipple reported that ampullary cancers are more likely to be of the adenomatous type with less general lymphatic and blood vessel invasion [11]. Current histopathological studies have also suggested further subdivision of ampullary carcinomas based on their exact histopathological findings [12, 13]. For example, intestinal ampullary adenocarcinomas arise from the surrounding intestinal epithelial layer, whereas pancreatobiliary ampullary cancers originate in the endothelium of the distal bile duct or pancreatic duct $[14,15]$.

Computed tomography $(\mathrm{CT})$ and magnetic resonance imaging cholangiopancreatography (MRCP) constitute the current clinical diagnostic methods of choice. Other methods, such as endosonography and endoscopic retrograde cholangiopancreatography (ERCP), allow for sample collection and thus permit further histological differentiation. A radical pancreaticoduodenectomy, performed either as a pylorus-preserving pancreatic head resection (PPPD) or a classic Whipple procedure (KW), is considered to be the gold standard therapy for ampullary carcinoma. Currently, endoscopic papillectomy is increasingly performed as an initial intervention in suspected benign papillary tumors [16, 17]. The decision to perform a subsequent pancreaticoduodenectomy may be based on the histopathological finding of the resected specimen. The resectability of ampullary carcinoma with a curative intention is $76.5 \%$ to $89.4 \%[2,18]$. Due to the rarity of this tumor, studies describing the longterm progress are scarce and are available only for up to 10 years after resection. Overall long-term survival still remains low. A major component in this issue is tumor recurrence. The aim of this retrospective study was to identify factors that influence the long-term survival in a large patient population over 15 years.

\section{Patients and Methods}

2.1. Preoperative Data. Between 1992 and 2007, 143 patients underwent resection of histologically verified ampullary cancer at our institution. Of these patients, 87 (61\%) were men and $56(39 \%)$ were women, with a median age of 64 (33-83) years. The median body mass index (BMI) in the patient group was $24.8(13.5-38.8) \mathrm{kg} / \mathrm{m}^{2}$. Forty-three (30\%) patients presented with a Karnofsky index below $80 \%$. Nicotine consumption was noted in $44(31 \%)$ patients and regular alcohol consumption in $32(22 \%)$ patients. Preoperative symptoms were apparent in 130 (91\%) patients. Seventyfive (52\%) patients presented with jaundice, and $88(62 \%)$ patients had nonspecific epigastric pain. Twenty-nine (20\%) patients described a weight loss of more than $10 \mathrm{~kg}$ in the three months preceding the presentation. Permanent nausea affected $29(20 \%)$ patients, and a reduced performance status was experienced by 27 (19\%) patients (Table 1). Twenty-one (15\%) patients already presented with diabetes mellitus, of whom $15(10 \%)$ were insulin-dependent and $6(4 \%)$ were on
TABLE 1: Characteristics of the patients.

\begin{tabular}{lc}
\hline $\begin{array}{l}\text { Number of patients } \\
\text { Gender }\end{array}$ & $n=143$ \\
$0^{\star}$ & $87(61 \%)$ \\
$\stackrel{+}{\text { Median age: years (range) }}$ & $56(39 \%)$ \\
Median body mass index (range) & $64(33-83)$ \\
Preoperative symptoms & $24.8(13.5-38.8)$ \\
Jaundice & \\
Nonspecific epigastric pain & $75(52 \%)$ \\
10\% reduction of body weight & $88(62 \%)$ \\
Nausea & $29(20 \%)$ \\
Reduced performance status & $29(20 \%)$ \\
Incidental finding & $27(19 \%)$ \\
\hline
\end{tabular}

oral antidiabetics. Thirteen (9\%) patients had a history of pancreatitis. In the context of diagnosis, 121 (85\%) patients had an abdominal CT, and a tumor was diagnosed in 56 cases ( $46 \%$ of all CT examinations). An endosonography was performed in $34(24 \%)$ patients, with tumor findings in 22 (65\% of all endosonographies) patients. Preoperative endoscopic retrograde cholangiography (ERC) was performed in 131 patients (92\%), with evidence of tumor in 105 patients (73\%). A papillotomy was undertaken in 57 patients (40\%), and preoperative stent placement in the common bile duct was performed in 39 patients (27\%). Preoperative laboratory chemical examinations gave a median CA 19-9 value of $23 \mathrm{U} / \mathrm{L}$ (1-9171), a bilirubin level of $1.7 \mathrm{mg} / \mathrm{dL}$ (0.2-44.4), and a $\gamma \mathrm{GT}$ of $172 \mathrm{U} / \mathrm{L}(6-1865)$.

2.2. Surgical Procedure. In $86(60 \%)$ patients a pyloruspreserving pancreaticoduodenectomy (PPPD) was performed and in 57 (40\%) patients a Kausch-Whipple pancreaticoduodenectomy (KW) was performed. Pancreatoenteral anastomosis was performed as pancreatico jejunostomy in 123 patients (86\%) or pancreaticogastrostomy in 20 patients (14\%) using a mattress suture technique in 98 patients (69\%) and Cattell duct-to-mucosa technique in 45 patients (31\%). Due to tumor infiltration, partial portal vein resection has been performed in two (1\%) patients. Reconstruction of the superior mesenteric artery was indicated in one patient (1\%). The median operation time was 325 (182-785) minutes, with an average blood loss of $500 \mathrm{~mL}(100-3000)$. A total of 43 (30\%) patients were intraoperatively substituted with packed red blood cells (PRBC). Pancreatic reconstruction was performed in 132 (92\%) patients as pancreaticojejunostomy (PJ) and in 11 patients as pancreaticogastrostomy (PG) (8\%). The operation was extended in nine (6\%) patients, with four patients receiving a partial liver resection, two a splenectomy and partial colon resection, and one a nephrectomy. Intraoperative complications occurred in 4 (3\%) patients; three patients had bleeding that was difficult to control and one patient experienced both myocardial infarction and cardiac arrhythmia. All of the operations were performed in line with tumor-surgical criteria by experienced visceral 
surgeons who were taking a curative approach. Both PPPD and KW were performed in accordance with international standards as en bloc dissection with lymphadenectomy along the hepatoduodenal ligament, celiac trunk, and superior mesenteric artery. The resection areas were classified intraoperatively as curative (R0) when no microscopic evidence of tumor cells was present histopathologically. The tumor stage was graded using the UICC classification of 2009 for ampullary cancers [19].

2.3. Standard Postoperative Care. Every patient received a nasogastric tube for gastric decompression. Amylase and/or lipase levels were monitored daily in the serum and in the intraoperatively placed abdominal drains (Degania Silicone Europe $\mathrm{GmbH}$, Regensburg, Germany) on the first and fourth postoperative days. Radiological contrast imaging was performed on the fifth postoperative day over the nasogastric tube.

The diagnosis of a postoperative pancreatic fistula formation (POPF) was based on the definition of the International Study Group on Pancreatic Fistula (ISGPF) [20]. The levels of amylase in the intraoperatively placed drains were not available for all subjects in our database. The lipase levels in the drains had always been measured. We therefore slightly modified the ISGPF definitions and used amylase or lipase levels in the drains to define the existence of a POPF. Postpancreatectomy hemorrhage ( $\mathrm{PPH})$ and delayed gastric emptying (DGE) were also defined based on the International Study Group of Pancreatic Surgery (ISGPS) definitions. [21, 22]. However, the definitions of ISGPS for POPF, PPH, and DGE were not published until 2004 and 2007, respectively. Thus, incidences of POPF and PPH had to be retrospectively evaluated.

2.4. Statistics. The data were collected in a database (Microsoft Access 2.0, Microsoft Corporation, Seattle, USA) and evaluated retrospectively. Unless otherwise specified, the data are expressed as median and range. Survival analysis was determined by means of the Kaplan-Meier method (log-rank test) and specific risk factors by the Mann-Whitney $U$ test using SPSS for Windows 14.0 (SPSS Inc. Chicago, IL, USA). A $P$ value below 0.05 was considered to be significant.

\section{Results}

3.1. Postoperative Progress and Surgical Complications. The median length of hospital stay was 16 (9-100) days. The median stay in intensive care was 3 (1-74) days. Twelve (8\%) patients developed POPF requiring operative revision in five cases. Insufficiency of the bile duct anastomosis occurred in two (1\%) patients. In total, revision surgery was undertaken in $10(7 \%)$ patients (Table 2). These revisions comprised four residual pancreatectomies and one new installation of the pancreatoenteral anastomosis (a pancreaticogastrostomy was followed by a pancreaticojejunostomy) and three revisions for wound dehiscence and two instances of PPH. Postoperative delayed gastric emptying occurred in 8 patients $(6 \%)$. The perioperative lethality was $3.5 \%$. The cause of death
TABLE 2: Operative and postoperative course.

\begin{tabular}{lc}
\hline Median operation time (minutes/range) & $325(182-785)$ \\
Median intraoperative blood loss (mL/range) & $500(100-3000)$ \\
Intraoperative complications & $4(3 \%)$ \\
Postoperative complications & $34(24 \%)$ \\
$\quad$ Wound infection & $14(10 \%)$ \\
$\quad$ Postpancreatectomy hemorrhage (PPH) & $6(4 \%)$ \\
$\quad$ Postoperative pancreatic fistula (POPF) & $12(8 \%)$ \\
$\quad$ Bile leak & $2(1 \%)$ \\
$\quad$ Delayed gastric emptying (DGE) & $8(6 \%)$ \\
Reoperation & $10(7 \%)$ \\
In-hospital mortality & $5(3.5 \%)$ \\
\hline
\end{tabular}

was sepsis in two patients, and one patient had surgically untreatable bleeding, cardiac decompensation from known cardiac insufficiency, or acute myocardial infarction. Within the observation period, 18 (13\%) patients underwent inpatient readmission. Of these, 10 (7\%) patients were operated on again for reasons unrelated to the underlying condition. Emerging diabetes mellitus was diagnosed in $9(6 \%)$ patients, and $64(45 \%)$ patients needed postoperative enzyme substitution at mealtimes. The total mortality was $69 \%$ in a median postoperative observation period of 30 months (0-205).

3.2. TNM. The histological examination of the pathological specimen and categorization by means of TNM classification resulted in a pTis stage in $2(1 \%)$ patients and a pT1 stage in $14(10 \%)$ patients. An almost identical number of patients had pT2 (53 patients; 37\%) and pT3 stages (54 patients; 38\%). In $20(14 \%)$ patients a pT4 stage was diagnosed. Positive lymph node involvement (pN1) was evident in 69 (48\%) cases. More than half of the patients were in a G2 stage (75 patients; $52 \%$ ) of differentiation (pG), followed by stage G3 in 36\% (52 patients). Fifteen (10\%) patients presented with a G1 stage and 1 (1\%) patient with a G4 stage. The tumor size was smaller than $2 \mathrm{~cm}$ in diameter in 53 patients (37\%) and bigger than $2 \mathrm{~cm}$ in 90 patients $(63 \%)$.

Microscopically detected tumor infiltration, detectable by microscopy (R1) of the resection margins or at the retropancreatic ablation level, was evidenced in 12 (8\%) patients. Lymphatic invasion was present in $70(49 \%)$ patients and vascular invasion in $17(12 \%)$ patients in the final histology.

3.3. UICC Stages. As a result of classifying the 143 patients as per the UICC stages, 16 (11\%) patients were stage la and 33 (23\%) patients were stage $1 b$. Stage $2 b$, with 51 patients (35\%), was the most frequent. In comparison, 20 patients (14\%) were stage $2 \mathrm{a}$ and $16(11 \%)$ and $7(6 \%)$ patients were stages 3 and 4 , respectively.

3.4. Survival and Prognostic Factors. After 1-, 5-, 10-, and 15year periods, the overall survival of the examined patient population was $79 \%, 40 \%, 25 \%$, and $10 \%$, respectively, with a median survival term of 37 months (Figure 1). Survival analysis (log-rank) resulted in a significantly reduced survival for patients who had a reduced general condition $(P=0.008)$, 
TABLE 3: Survival and prognostic factors with respect to survivalmultivariate analysis.

\begin{tabular}{lcc}
\hline & $P$ value & $\begin{array}{c}\text { Odds ratio } \\
\text { (95\% confidence } \\
\text { interval) }\end{array}$ \\
\hline $\begin{array}{l}\text { No lymphatic invasion } \\
\begin{array}{l}\text { No intraoperative } \\
\text { administration of PRBC }\end{array}\end{array}$ & $P=0.000$ & $0.248(0.145-0.425)$ \\
$\begin{array}{l}\text { Preoperatively elevated } \\
\text { CA 19-9 }\end{array}$ & $P=0.008$ & $0.510(0.311-0.836)$ \\
\hline
\end{tabular}

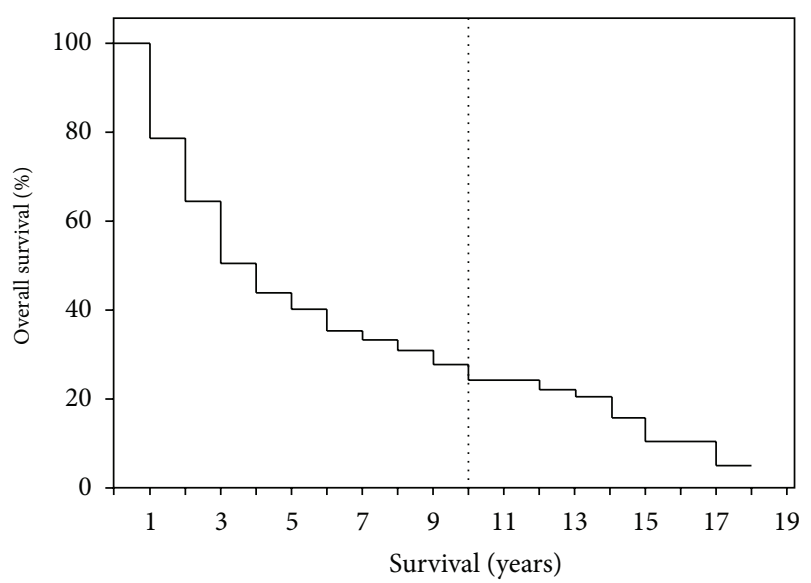

FIGURE 1: The overall survival for patients after the resection of ampullary carcinoma with curative intention.

required intraoperative administration of $\operatorname{PRBC}(P=0.003)$, had POPF $(P=0.013)$, had an advanced tumor stage $(P=0.0001)$ (Figure 2$)$, had a pT4 tumor invasion depth $(P=0.0001)$, had a positive lymph node stage $(0.0001)$ (Figure 3), had a pG4 tumor grade $(P=0.0001)$, had a microscopically or macroscopically positive resection margin $(P=0.02)$ (Figure 4$)$, had vascular $(P=0.008)$ or lymphatic invasion $(P=0.0001)$ (Figure 5$)$, and had a preoperatively elevated CA $19-9(P=0.008)$. There were no significant differences in regard of overall survival in patients who received a PPPD and patients who underwent classic Whipple procedure $(P=0.222)$. A tumor size smaller than $2 \mathrm{~cm}$ did not have a significant effect on overall survival $(P=0.458)$. Examining the risk factors with respect to survival, multivariate analysis revealed that the following are risk factors for poor prognosis: lymphatic invasion $(P=$ $0.000)$, intraoperative administration of PRBC $(P=0.008)$, and a preoperatively elevated CA 19-9 $(P=0.023)$ (Table 3$)$.

\section{Discussion}

In 1912, Hirschel conducted the first documented singlestage resection of an ampullary carcinoma in Heidelberg, Germany [23]. Since then, morbidity and mortality have been reduced continuously through modifications of the operative procedure and through general progress in diagnosis and peri- and postoperative management. However, long-term

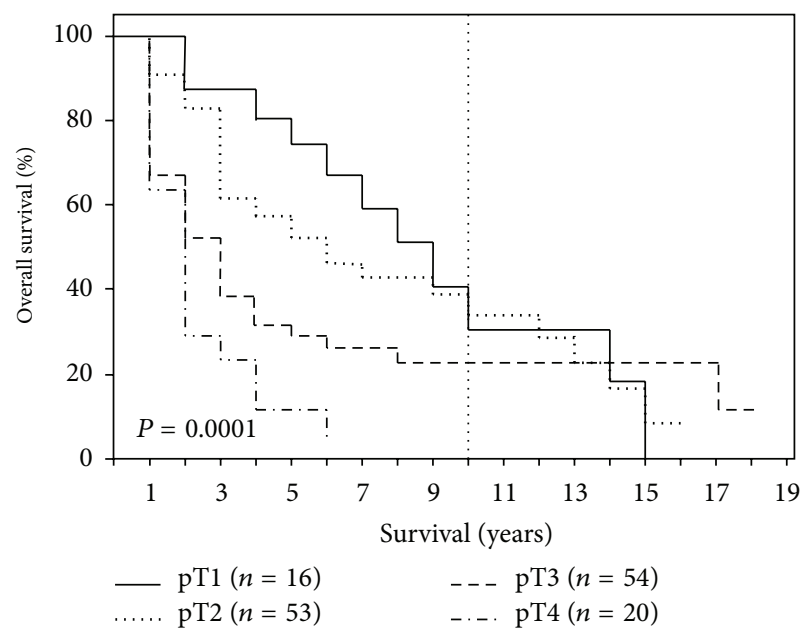

FIGURE 2: Survival depending on tumor stage (pT1, pT2, pT3, and pT4).

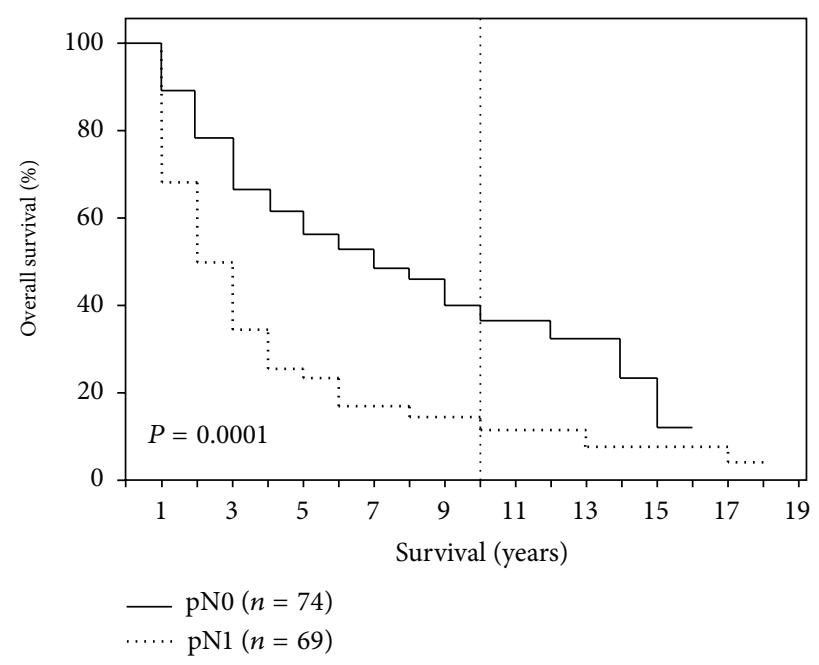

FIgURE 3: Survival according to lymph node status (pN0 versus pN1).

survival following curative resection of ampullary carcinoma remains low. The reported 5-year survival rates vary from 30 to $70 \%[4,24-28]$. In our study, the 5-year survival equaled $40 \%$ which is equivalent to the results of a retrospective study of a large American patient population by O'Connel et al. who reported a 5-year survival of $36.8 \%$ for a total of 3292 patients, however only 1301 of whom (40\%) underwent primary surgical therapy [29]. The majority of current studies looking into the long-term follow-up of ampullary cancer are conducted multicentrically and examine the long-term results for up to a maximum of ten years following resection. The information on factors that influence the long-term prognosis following the resection of ampullary carcinomas is therefore limited. It should be noted that ampullary cancer does not occur frequently overall but constitutes a relevant proportion (20$40 \%$ ) of all resected tumors of the periampullary region [2-4]. One reason for this situation is the high rate of resectability at the time of diagnosis, specified in the literature as up to 


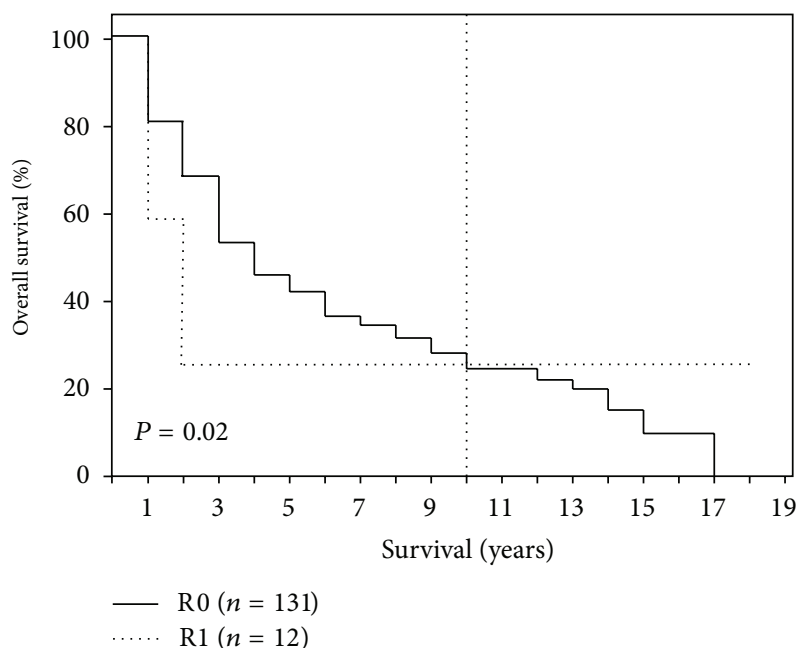

FIGURE 4: Survival depending on surgical radicality (R0 versus R1).

$80 \%$ which is significantly higher than for pancreatic head carcinoma $(20 \%)[3,30]$. The anatomical location and the exophytic growth pattern of ampullary carcinomas, leading to early occlusion of the bile duct and therefore often-early clinical diagnosis, explain the high resectability rate and the positive results relative to the surgical radicality of the procedure [4-6]. This is underlined by the results of our study in which a total of 131 patients (92\%) received $\mathrm{R} 0$ resection of the primary tumor with a 10 -year survival of $25 \%$ and 15 -year survival of $10 \%$, respectively. In the survival analysis (log-rank), our study identified the following prognostic factors that were accompanied by a significantly reduced long-term survival: reduced general condition at the time of surgery, intraoperative administration of PRBC, POPF, tumor stage, substantial invasion depth of the tumor, lymph node stage, histological grading, resection border, vascular and lymphatic vessel invasion, and CA 19-9 levels higher than $37 \mathrm{U} / \mathrm{L}$. There were no significant differences in regard of overall survival between patients who underwent PPPD in comparison to the classic Whipple procedure. A postpyloric resection approach therefore appears to be safe in patients with ampullary cancer.

In the multivariate analysis lymphatic vessel invasion, intraoperative administration of PRBCs, and an elevated CA 19-9 level were identified as independent risk factors for a reduced long-term survival. There is consensus in the literature for most carcinomas of the gastrointestinal tract (esophagus, stomach, and colorectum) regarding the influence of lymph node status on long-term prognosis. Aside from the results of our study, this hypothesis is substantiated by the results of Hurtuk et al., who noted the significant influence of positive lymph node involvement on long-term survival especially for ampullary and pancreatic carcinoma [31]. The 5-year survival reported in the literature is 0 to $30 \%$ when there is positive lymph node involvement and 39 to $78 \%$ in patients who lack lymph node involvement [32]. There is controversy over the extent of the lymphadenectomy. At our clinic, partial pancreaticoduodenectomy is performed

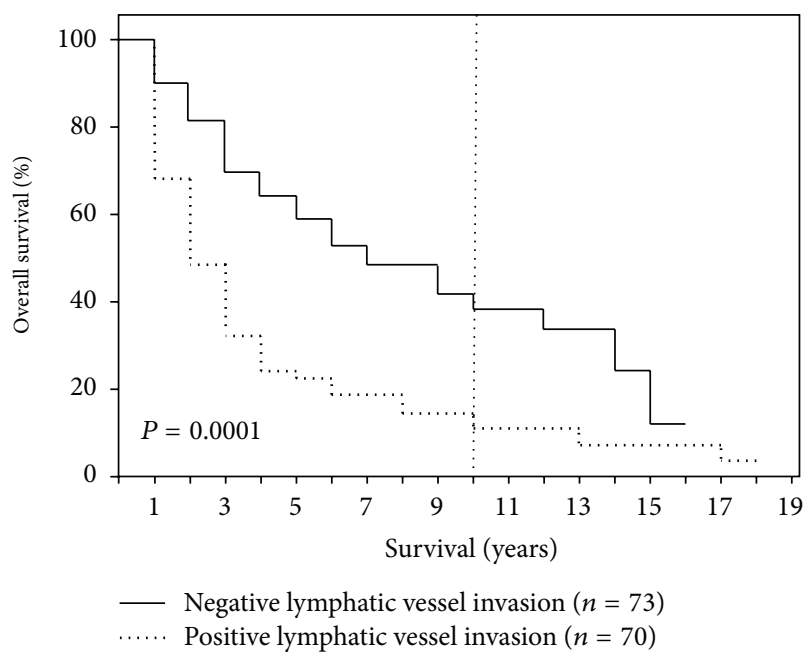

FIGURE 5: Survival depending on lymphatic vessel invasion (L0 versus L1).

with a comprehensive lymphadenectomy, along the hepatoduodenal ligament, celiac trunk, and superior mesenteric artery. However, in a study in which the results from standard lymphadenectomy and extended lymphadenectomy were compared, there were no significant differences with respect to long-term survival (56 versus 60\%) [33].

Beyond radical surgical approaches, also endoscopic treatment options for ampullary tumors exist such as endoscopic resection [34], photodynamic therapy [35], and electrofulguration [36]. Endoscopic resection can provide a safe and effective treatment option for benign ampullary tumors. If an ampullary tumor appears to be benign and the biopsy samples are negative for malignancy, endoscopic papillectomy should be considered as an initial intervention. The decision on whether to perform a subsequent pancreaticoduodenectomy should then be based on the histopathological findings. However, false negative rates of 40 to $85 \%$ have been reported for endoscopic biopsies and small ampullary cancers may be missed [37]. According to the results of our study, a tumor size smaller than $2 \mathrm{~cm}$ even in early tumor stages does not correlate with an improved survival. A delayed treatment may therefore be fatal. An accurate differentiation between benign and malignant lesions can only be achieved by radical pancreaticoduodenectomy. Prospective randomized trials will have to evaluate a possible benefit of endoscopic resection for small ampullary malignancies, for example, in patients with an increased operative risk score.

Howe et al. also identified lymph node metastases and a positive tumor cutting margin as risk factors for reduced long-term survival [4]. However, at the same time, these authors subdivided ampullary carcinomas into two subgroups by way of detailed histopathology and found that median survival of those tumors with a histologically verified pancreatobiliary origin was significantly lower in comparison to tumors with a histologically verified intestinal origin (22 months versus 60 months) [4]. Outerbridge reported back in 1913 that ampullary cancer could exhibit different 
histological origins [38]. Apart from origins in the duodenal mucosa, the epithelia of the common pancreatobiliary ductal system, pancreatic duct, or bile duct are possible points of origin. In clinical practice, due to the heterogeneity of the tumor and frequent, simultaneously present preneoplastic lesions, the exact histological origin can often be not clearly differentiated. In our study, we did not further classify ampullary carcinomas based on detailed histological findings. Kimura et al. (1994), who examined 53 patients with ampullary carcinoma, were one of the first to describe histological criteria that allow for more accurate allocation to either pancreatobiliary or intestinal origin [39]. These authors also reported that ampullary cancers of pancreatobiliary origin are more frequently accompanied by lymph node involvement and had a worse prognosis than carcinomas of an intestinal origin [39]. Zhou et al. reported in 2004 the use of cytokeratin and apomycin markers to assign ampullary cancers histologically and unambiguously to one of the two subgroups [13]. However, these methods are still not a popular standard today, even though Westgaard et al. showed that the histological subtype is an essentially more relevant prognostic factor than the otherwise typical affiliation to one of the anatomical subtypes of periampullary carcinomas [40].

Tumor recurrence after resection with curative intention remains a key problem in the long-term prognosis following radical resection. The tumor recurrence rate, as reported in the literature, varies from 28 to $44 \%$. Examples of key manifestation regions are the liver and aortocaval lymph node metastases, as well as locoregional tumor recurrence $[18,26,41]$. Postoperative adjuvant chemotherapy [42] and radiochemotherapy $[43,44]$ have been shown to improve survival outcomes in patients with periampullary carcinomas. In current studies, however, patients considered for postoperative adjuvant therapy often had adverse prognostic factors, such as positive lymph node involvement, higher tumor stage, or poor tumor differentiation, compared with patients who were treated with surgery alone [45]. According to the results of our study, adjuvant therapy should be recommended for patients with positive tumor cutting margins or other risk constellations, such as lymph node involvement, tumor invasion into the surrounding tissue, or poorly differentiated tumor grade. However, there is an urgent need for further studies on the influence of adjuvant therapy on the long-term prognosis after the resection of ampullary cancer.

There are several limitations to the present study. Although the clinical data were prospectively collected, the study design and analysis are retrospective and are therefore subject to an inherent selection bias. Moreover, due to the rarity of this tumor entity, in an attempt to achieve a statistically relevant patient cohort, the patients included in this study were treated over a time period of 15 years. During this time surgical techniques, peri- and postoperative management, and the role of adjuvant therapy were not consistent with recent recommendations. However, to the best of our knowledge, this study is the first to investigate the factors that influence the long-term survival in a large patient population over a period of 15 years after the surgical treatment of ampullary carcinomas. This study may therefore help to guide future practice patterns and treatment recommendations.

\section{Conclusion}

The prognosis of ampullary carcinoma is clearly better than that of other carcinomas of the periampullary region. Nevertheless, the results of our study show that factors such as an increased tumor stage, considerable invasion depth of the tumor, positive lymph node involvement, blood vessel and lymphatic invasion of the tumor, and a CA 19-9 level higher than $37 \mathrm{U} / \mathrm{L}$ are accompanied by a reduced long-term prognosis. Subsequent adjuvant therapy remains essential especially in patients with this constellation of risk factors. However, additional studies are necessary to specify the role of adjuvant therapy in improving long-term results after the resection of ampullary carcinomas.

\section{Conflict of Interests}

The authors have no conflict of interests or financial ties to disclose.

\section{Authors' Contribution}

Fritz Klein collected the data and wrote the paper. Dietmar Jacob designed and performed the research. Marcus Bahra collected the data. Gero Puhl collected the data. Andreas Andreou collected the data. Safak Gül collected the data. Olaf Guckelberger designed and performed the research. All of the authors contributed to the design and interpretation of the study and to further drafts.

\section{References}

[1] K. Yamaguchi and M. Enjoji, "Carcinoma of the ampulla of Vater. A clinicopathologic study and pathologic staging of 109 cases of carcinoma and 5 cases of adenoma," Cancer, vol. 59, no. 3, pp. 506-515, 1987.

[2] A. Nakase, Y. Matsumoto, K. Uchida, and I. Honjo, "Surgical treatment of cancer of the pancreas and the periampullary region: cumulative results in 57 institutions in Japan," Annals of Surgery, vol. 185, no. 1, pp. 52-57, 1977.

[3] C. J. Yeo, T. A. Sohn, J. L. Cameron, R. H. Hruban, K. D. Lillemoe, and H. A. Pitt, "Periampullary adenocarcinoma: analysis of 5-year survivors," Annals of Surgery, vol. 227, no. 6, pp. 821-831, 1998.

[4] J. R. Howe, D. S. Klimstra, R. D. Moccia, K. C. Conlon, and M. F. Brennan, "Factors predictive of survival in ampullary carcinoma," Annals of Surgery, vol. 228, no. 1, pp. 87-94, 1998.

[5] C. G. Willett, A. L. Warshaw, K. Convery, and C. C. Compton, "Patterns of failure after pancreaticoduodenectomy for ampullary carcinoma," Surgery Gynecology and Obstetrics, vol. 176, no. 1, pp. 33-38, 1993.

[6] P. Bucher, G. Chassot, Y. Durmishi, F. Ris, and P. Morel, "Longterm results of surgical treatment of Vater's ampulla neoplasms," Hepato-Gastroenterology, vol. 54, no. 76, pp. 1239-1242, 2007.

[7] K. Sahora, I. Kührer, D. Trenkwitz et al., "Pankreaskarzinom und periampulläres Karzinom," Journal für Gastroenterologische und Hepatologische Erkrankungen, vol. 7, no. 2, pp. 36-44, 2009.

[8] B. W. Miedema, M. G. Sarr, J. A. van Heerden et al., "Complications following pancreaticoduodenectomy: current management," Archives of Surgery, vol. 127, no. 8, pp. 945-950, 1992. 
[9] K. W. Warren, D. S. Choe, J. Plaza, and M. Relihan, "Results of radical resection for periampullary cancer," Annals of Surgery, vol. 181, no. 5, pp. 534-540, 1975.

[10] F. Michelassi, F. Erroi, P. J. Dawson et al., "Experience with 647 consecutive tumors of the duodenum, ampulla, head of the pancreas, and distal common bile duct," Annals of Surgery, vol. 210, no. 4, pp. 544-556, 1989.

[11] A. O. Whipple, "A reminiscence: pancreaticduodenectomy," Review of Surgery, vol. 20, pp. 221-225, 1963.

[12] W. Kimura and K. Ohtsubo, "Incidence, sites of origin, and immunohistochemical and histochemical characteristics of atypical epithelium and minute carcinoma of the papilla of Vater," Cancer, vol. 61, no. 7, pp. 1394-1402, 1988.

[13] H. Zhou, N. Schaefer, M. Wolff, and H. P. Fischer, "Carcinoma of the ampulla of vater: comparative histologic/immunohistochemical classification and follow-up," The American Journal of Surgical Pathology, vol. 28, no. 7, pp. 875-882, 2004.

[14] K. Baczako, M. Büchler, H. G. Beger, C. J. Kirkpatrick, and O. Haferkamp, "Morphogenesis and possible precursor lesions of invasive carcinoma of the papilla of Vater: epithelial dysplasia and adenoma," Human Pathology, vol. 16, no. 3, pp. 305-310, 1985.

[15] I. C. Talbot, J. P. Neoptolemos, D. E. Shaw, and D. Carr-Locke, "The histopathology and staging of carcinoma of the ampulla of vater," Histopathology, vol. 12, no. 2, pp. 155-165, 1988.

[16] G. C. Harewood, N. L. Pochron, and C. J. Gostout, "Prospective, randomized, controlled trial of prophylactic pancreatic stent placement for endoscopic snare excision of the duodenal ampulla," Gastrointestinal Endoscopy, vol. 62, no. 3, pp. 367-370, 2005.

[17] S. Seewald, S. Omar, and N. Soehendra, "Endoscopic resection of tumors of the ampulla of Vater: how far up and how deep down can we go?" Gastrointestinal Endoscopy, vol. 63, no. 6, pp. 789-791, 2006.

[18] T. Todoroki, N. Koike, Y. Morishita et al., "Patterns and predictors of failure after curative resections of carcinoma of the ampulla of vater," Annals of Surgical Oncology, vol. 10, no. 10, pp. 1176-1183, 2003.

[19] L. H. Sobin, M. K. Gospodarowicz, C. Wittekind, and International Union against Cancer, TNM Classification of Malignant Tumours, Wiley, Blackwell, 7th edition, 2009.

[20] C. Bassi, G. Butturini, E. Molinari et al., "Pancreatic fistula rate after pancreatic resection: the importance of definitions," Digestive Surgery, vol. 21, no. 1, pp. 54-59, 2004.

[21] M. N. Wente, J. A. Veit, C. Bassi et al., "Postpancreatectomy hemorrhage (PPH) - an international study group of pancreatic surgery (ISGPS) definition," Surgery, vol. 142, no. 1, pp. 20-25, 2007.

[22] M. N. Wente, C. Bassi, C. Dervenis et al., "Delayed gastric emptying (DGE) after pancreatic surgery: a suggested definition by the international study group of pancreatic surgery (ISGPS)," Surgery, vol. 142, no. 5, pp. 761-768, 2007.

[23] D. W. Crist and J. L. Cameron, "The current status of the Whipple operation for periampullary carcinoma," Advances in Surgery, vol. 25, pp. 21-49, 1992.

[24] M. A. Talamini, R. C. Moesinger, H. A. Pitt et al., "Adenocarcinoma of the ampulla of Vater: a 28-year experience," Annals of Surgery, vol. 225, no. 5, pp. 590-600, 1997.

[25] J. P. Duffy, O. J. Hines, J. H. Liu et al., "Improved survival for adenocarcinoma of the ampulla of Vater: fifty-five consecutive resections," Archives of Surgery, vol. 138, no. 9, pp. 941-950, 2003.
[26] V. Bettschart, M. Q. Rahman, F. J. F. Engelken, K. K. Madhavan, R. W. Parks, and O. J. Garden, "Presentation, treatment and outcome in patients with ampullary tumours," The British Journal of Surgery, vol. 91, no. 12, pp. 1600-1607, 2004.

[27] A. Di Giorgio, S. Alfieri, F. Rotondi et al., "Pancreatoduodenectomy for tumors of Vater's ampulla: report on 94 consecutive patients," World Journal of Surgery, vol. 29, no. 4, pp. 513-518, 2005.

[28] K. M. Brown, A. J. Tompkins, S. Yong, G. V. Aranha, and M. Shoup, "Pancreaticoduodenectomy is curative in the majority of patients with node-negative ampullary cancer," Archives of Surgery, vol. 140, no. 6, pp. 529-533, 2005.

[29] J. B. O’Connell, M. A. Maggard, J. Manunga Jr. et al., "Survival after resection of ampullary carcinoma: a national populationbased study," Annals of Surgical Oncology, vol. 15, no. 7, pp. 18201827, 2008.

[30] R. D. Kim, P. S. Kundhal, I. D. McGilvray et al., "Predictors of failure after pancreaticoduodenectomy for ampullary carcinoma," Journal of the American College of Surgeons, vol. 202, no. 1, pp. 112-119, 2006.

[31] M. G. Hurtuk, C. Hughes, M. Shoup, and G. V. Aranha, “Does lymph node ratio impact survival in resected periampullary malignancies?" The American Journal of Surgery, vol. 197, no. 3, pp. 348-352, 2009.

[32] H. P. Hsu, Y. S. Shan, Y. H. Hsieh, T. M. Yang, and P. W. Lin, "Predictors of recurrence after pancreaticoduodenectomy in ampullary cancer: comparison between non-, early and late recurrence," Journal of the Formosan Medical Association, vol. 106, no. 6, pp. 432-443, 2007.

[33] C. J. Yeo, J. L. Cameron, K. D. Lillemoe et al., "Pancreaticoduodenectomy with or without distal gastrectomy and extended retroperitoneal lymphadenectomy for periampullary adenocarcinoma-part 2: randomized controlled trial evaluating survival, morbidity, and mortality," Annals of Surgery, vol. 236, no. 3, pp. 355-368, 2002.

[34] K. F. Binmoeller, S. Boaventura, K. Ramsperger, and N. Soehendra, "Endoscopic snare excision of benign adenomas of the papilla of Vater," Gastrointestinal Endoscopy, vol. 39, no. 2, pp. 127-131, 1993.

[35] R. Lambert, T. Ponchon, A. Chavaillon, and F. Berger, "Laser treatment of tumors of the papilla of Vater," Endoscopy, vol. 20, no. 1, pp. 227-231, 1988.

[36] E. Shemesh, S. Nass, and A. Czerniak, "Endoscopic sphincterotomy and endoscopic fulguration in the management of adenoma of the papilla of Vater," Surgery Gynecology and Obstetrics, vol. 169, no. 5, pp. 445-448, 1989.

[37] M. K. Jung, C. M. Cho, S. Y. Park et al., "Endoscopic resection of ampullary neoplasms: a single-center experience," Surgical Endoscopy, vol. 23, no. 11, pp. 2568-2574, 2009.

[38] G. Outerbridge, "Carcinoma of the papilla of Vater," Surgery, vol. 57, pp. 402-426, 1913.

[39] W. Kimura, N. Futakawa, S. Yamagata et al., "Different clinicopathologic findings in two histologic types of carcinoma of papilla of Vater," Japanese Journal of Cancer Research, vol. 85, no. 2, pp. 161-166, 1994.

[40] A. Westgaard, S. Tafjord, I. N. Farstad et al., "Pancreatobiliary versus intestinal histologic type of differentiation is an independent prognostic factor in resected periampullary adenocarcinoma," BMC Cancer, vol. 8, article 170, 2008.

[41] M. Kayahara, T. Nagakawa, T. Ohta, H. Kitagawa, and I. Miyazaki, "Surgical strategy for carcinoma of the papilla of 
Vater on the basis of lymphatic spread and mode of recurrence," Surgery, vol. 121, no. 6, pp. 611-617, 1997.

[42] H. Oettle, S. Post, P. Neuhaus et al., "Adjuvant chemotherapy with gemcitabine vs observation in patients undergoing curative-intent resection of pancreatic cancer: a randomized controlled trial," The Journal of the American Medical Association, vol. 297, no. 3, pp. 267-277, 2007.

[43] S. S. Sikora, P. Balachandran, K. Dimri et al., "Adjuvant chemoradiotherapy in ampullary cancers," European Journal of Surgical Oncology, vol. 31, no. 2, pp. 158-163, 2005.

[44] J. H. Klinkenbijl, J. Jeekel, T. Sahmoud et al., "Adjuvant radiotherapy and 5-fluorouracil after curative resection of cancer of the pancreas and periampullary region: phase III trial of the EORTC gastrointestinal tract cancer cooperative group," Annals of Surgery, vol. 230, no. 6, pp. 776-784, 1999.

[45] S. Bhatia, R. C. Miller, M. G. Haddock, J. H. Donohue, and S. Krishnan, "Adjuvant therapy for ampullary carcinomas: the Mayo clinic experience," International Journal of Radiation Oncology Biology Physics, vol. 66, no. 2, pp. 514-519, 2006. 


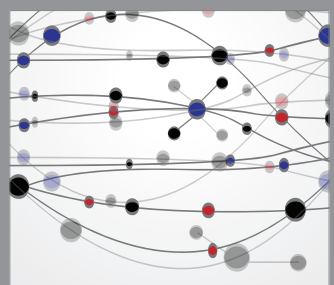

The Scientific World Journal
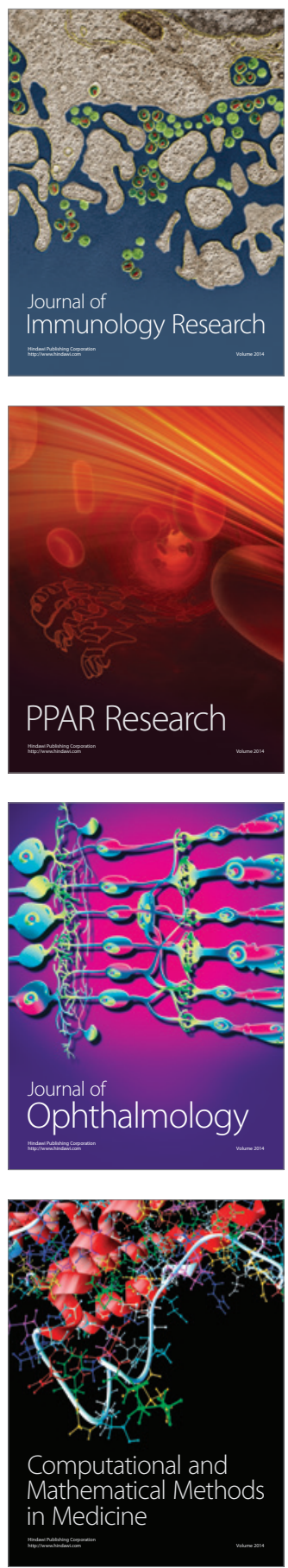

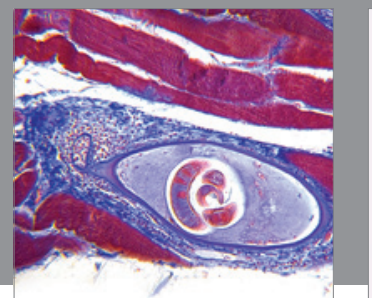

Gastroenterology

Research and Practice
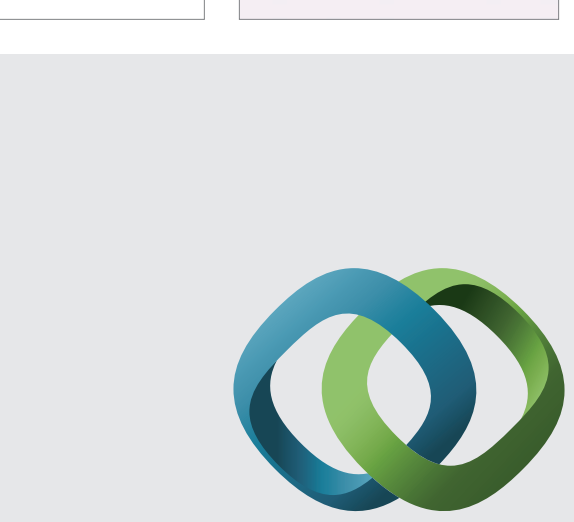

\section{Hindawi}

Submit your manuscripts at

http://www.hindawi.com
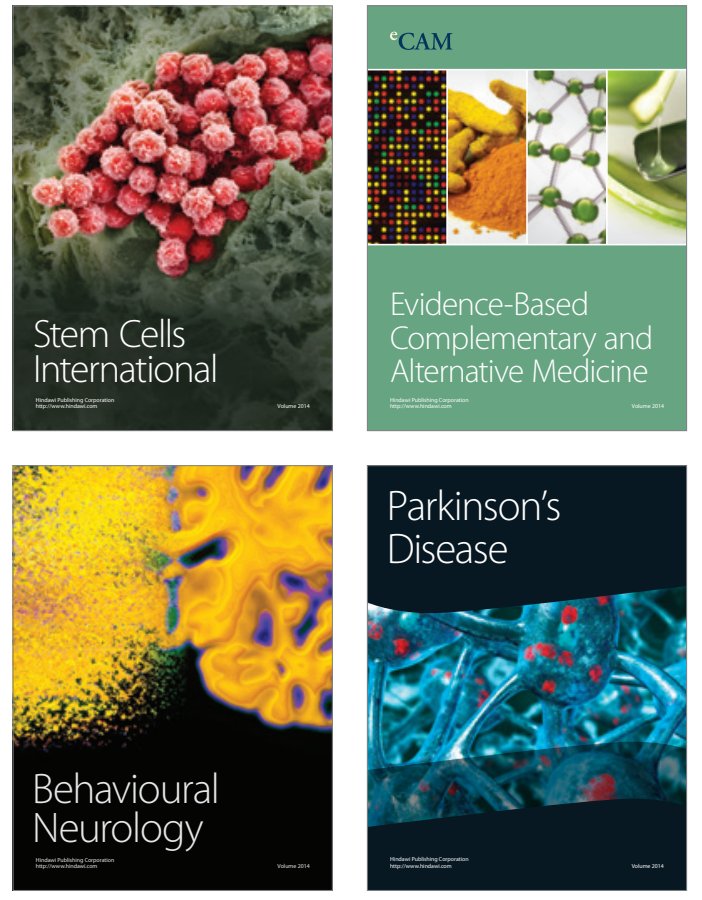
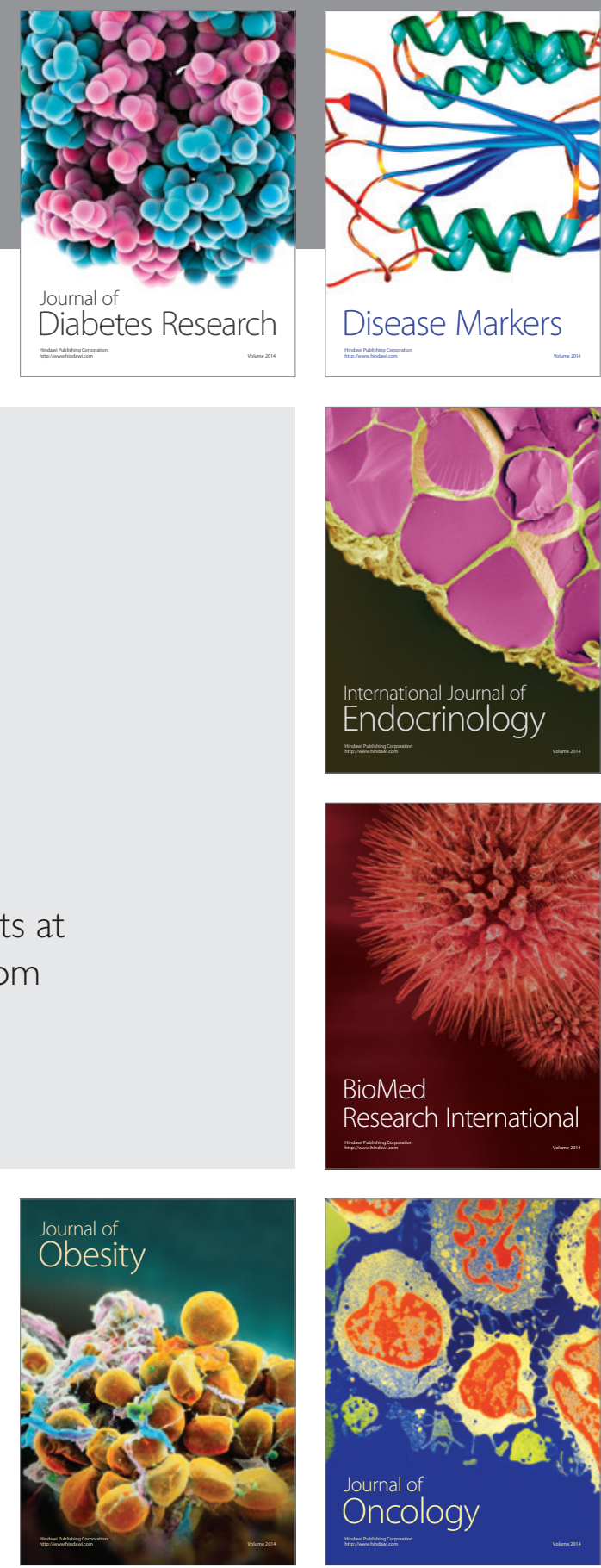

Disease Markers
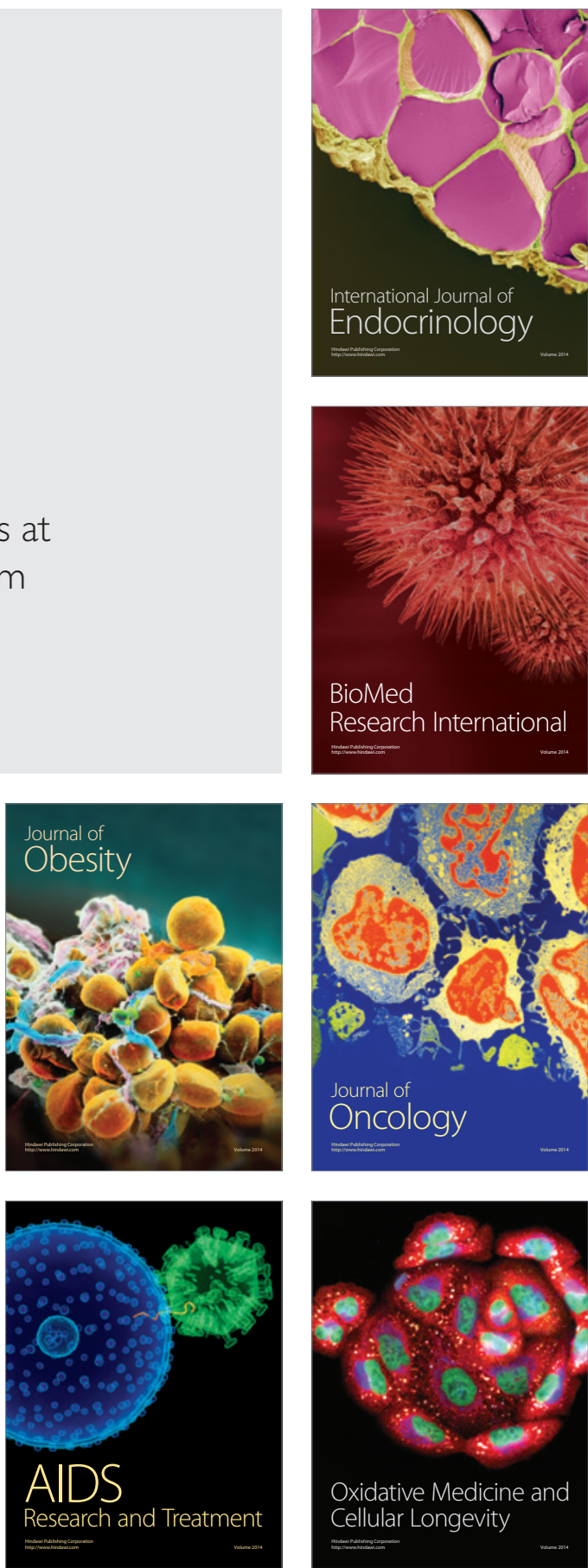